\title{
Estudio de calidad de información de los partes de interconsulta (P.I.C.) utilizados en las derivaciones de atención primaria a una unidad de salud mental
}

RESUMEN: El grado de cumplimentación correcta de los partes de interconsulta es un indicador de la calidad de la asistencia prestada.

PALABRAS CLAVE: Atención Primaria, Salud Mental, derivación, calidad.
ABSTRACT: The correct level of compliance of the interclinical notes is an index of the quality of health provision. KEY WORDS: Primary Care, Mental Health, referral, quality.

\section{Introducción.-}

La Salud Mental es un problema de gran importancia y prevalencia dentro de la población general. Los médicos de Atención Primaria ocupan un $40 \%$ de su tiempo en el manejo de las consultas psiquiátricas. En ocasiones es necesario hacer derivaciones al segundo nivel de Atención Especializada (Salud Mental). Este nivel es imprescindible para el abordaje de esta problemática, para apoyar y complementar la actuación de los médicos de familia y mejorar así la calidad asistencial (1). Dentro del modelo de Atención Primaria sólo es posible una atención a la salud de forma integrada y continuada, con la adecuada colaboración y comunicación entre ambos niveles $(2,3,4)$.

Ya en la mitad de los años 90 algunos autores señalaban la necesidad de consensuar procesos de derivación intercentros para ofrecer servicios de forma integrada (5).

Los facultativos de Atención Primaria utilizan principalmente los Partes de Interconsulta (PIC) escritos para derivar pacientes al segundo nivel asistencial, y por tanto también a las Unidades de Salud Mental, y establecer dicha comunicación. Su cumplimentación correcta se puede estudiar como expresión de la calidad de la asistencia prestada y como indicador de la capacitación técnica del profesional $(2,3)$. Existen otros medios (consulta telefónica...) para hacer la derivación que están infrautilizados (5).

Se ha venido observando que en estos PIC en ocasiones no aparecen datos que son fundamentales para la mejor atención de los pacientes. En los últimos años se ha 
visto que se van aportando mayor cantidad de estos datos, pero aún no resultan suficientes (6). Dentro de las informaciones que resultan básicas y de utilidad al especialista se encuentran, entre otras, la del motivo que lleva a solicitar la interconsulta así como la sospecha diagnóstica $(2,3)$.

Por todas estas razones, desde el Equipo de la Unidad de Salud Mental (U.S.M.) Emili Darder se propuso un estudio sobre la calidad de la información de los partes de interconsulta (PICS) enviados por los facultativos de Atención Primaria en la derivación de los pacientes a nuestra Unidad.

El objetivo de este trabajo es por lo tanto conocer cómo funcionan los cauces de información entre Atención Primaria y Atención Especializada (Salud Mental), evaluando la calidad de información de los partes de interconsulta procedentes de los médicos de Atención Primaria de los Centros de Salud derivantes a la U.S.M., es decir, el grado de cumplimentación correcta de la información clínico-administrativa que contienen dichas peticiones.

\section{Material y métodos}

Para la realización del trabajo se ha utilizado un diseño descriptivo de tipo transversal.

Los partes de Interconsulta son enviados por correo interno o fax a la USM Emili Darder, donde se llevó a cabo el estudio. Éstos son recogidos en un cajetín por la administrativa. Los PIC que conformaron la muestra fueron extraídos de este cajetín.

La muestra está formada por todas las interconsultas remitidas a la USM por correo desde mediados de marzo a mediados de abril de 2002. El total de los informes que conforman la muestra es de 201.

Han sido excluidos todos los PIC que eran remitidos desde otras entidades sanitarias: Hospital Son Dureta... así como las peticiones que llegaron por fax (que por otra parte son minoritarias), por la difícil legibilidad de las mismas.

La Unidad de Salud Mental Emili Darder atendía en el momento del estudio las peticiones de 8 centros de salud de su área geográfica: C.S.Pere Garau, C.S.Emili Darder, C.S.Son Gotleu, C.S.Coll d’en Rebassa, C.S.Son Ferriol, C.S.Llucmajor, C.S.Arenal, C.S.Trencadors, en total aproximadamente una población de 150.000 habitantes. El equipo lo forman 2 psiquiatras, 2 psicólogos a tiempo completo y uno a tiempo parcial, 2 enfermeros y una administrativa. Durante este año también ha formado parte del mismo la residente P.I.R., autora de este trabajo.

Se han recogido los datos de los PICs procedentes de estos 8 centros de salud, en el orden aleatorio en que iban llegando al centro.

No se comunicó a los equipos de Atención Primaria el hecho de que se estuvieran estudiando sus informes de Interconsulta, con el objetivo de no modificar sus hábitos, evitando así el efecto Hawthorne.

Se elaboró una hoja de recogida de datos de evaluación a cumplimentar por la autora del estudio, residente de Psicología Clínica (PIR-2) (figura 1). Ésta consta de dos apartados generales: datos formales y datos clínicos. Dentro del primero hay 18 ítems 
referentes a la identificación tanto del paciente como del médico derivante, así como otros datos de interés. En ambos casos se consigna también un apartado que hemos denominado "completo" y que se refiere a la aparición de todos los datos anteriores. En el segundo apartado, en 10 ítems, se hace referencia a los aspectos clínicos que es importante constatar a nuestro entender en el parte de interconsulta: enfermedad actual, antecedentes, tratamientos, diagnósticos, datos psicosociales del paciente y tipo de derivación realizada. Estos aspectos son definidos a continuación.

Entendemos por Enfermedad Actual la descripción de la sintomatología que presenta el paciente y que motiva la interconsulta.

Denominamos Motivo de la Interconsulta la razón que el facultativo especifica por la cual remite al paciente. Las opciones siguientes (valoración, derivación, tratamiento concreto, revisar tratamiento farmacológico) son algunas de las que puede hacer constar el médico como motivo de la interconsulta. En "Otros" están incluidas peticiones de nueva cita, cambio de centro, informes o por demanda propia del paciente.

Entendemos por Antecedentes Orgánicos, cualquier enfermedad somática o intervención quirúrgica que padezca o haya padecido el paciente y sea señalada en el PIC.

Los Tratamientos son los fármacos que el facultativo hace constar en la petición que el paciente toma para paliar estas enfermedades somáticas.

Los Antecedentes Psiquiátricos son los antecedentes personales de diagnósticos o tratamientos anteriores en el campo de la salud mental.

El Tratamiento prescrito en Atención Primaria se refiere a los psicofármacos que el facultativo de Atención Primaria ya ha pautado para abordar el trastorno o la sintomatología que presenta el paciente.

Observamos también si especifica la Duración de este tratamiento y la Respuesta, sea favorable o desfavorable, al mismo.

Diagnóstico Atención Primaria se refiere al diagnóstico que el médico de cabecera propone de lo que le ocurre al paciente.

Los Datos Psicosociales hacen referencia a la especificación por parte del facultativo de circunstancias de tipo familiar, económico, laboral o escolar, que puedan estar influyendo en el desarrollo y/o mantenimiento de los síntomas.

La Legibilidad se refiere a si la letra utilizada por el médico de cabecera es fácilmente decodificable a primera vista. Es un concepto subjetivo referente a la facilidad o dificultad para ser leída.

El Teléfono de Contacto se refiere el teléfono del centro de salud al cual habría que acudir en caso de clarificar información, etc.

El sistema utilizado en la recogida y codificación de datos es un sistema binario en el cual 1 significa presencia del dato en el impreso estudiado, que consta en el parte de interconsulta y 0 su ausencia, que no consta. En algunos ítems se añade el dígito 2 para señalar que no procede el dato de que se trate, en este caso por pertenecer a los PIC informatizados. 
INFORMES

Los datos han sido procesados mediante el programa SPSS y se han utilizado estadísticos de frecuencia y porcentajes para analizarlos.

\section{Resultados}

La muestra de PIC recogidos fue de 201 en total, procedentes de los 8 centros de salud arriba mencionados, con la siguiente distribución de frecuencias.(Tabla 1, figura 2).

Todos los centros de salud tienen un $100 \%$ de peticiones con un formato estándar(que consta de 4 hojas autocopiativas de color blanco, azul, amarillo y rosa), sólo el centro de salud de Son Gotleu presenta un 71,1\% de partes con un formato nuevo, informatizado y sin hojas autocopiativas.

En la Tabla 2 se ofrece un resumen de todos los resultados obtenidos para cada variable, que a continuación comentamos.

En cuanto a los Datos Formales podemos destacar, dentro de los relativos al paciente, que casi un $28 \%$ de los PIC no tienen todos los datos completos (nombre y apellidos, teléfono, número de identificación y edad), un 72,1\% sí. Es un porcentaje muy superior al 6-7\% del estudio de Huertas (2).

En un $15,4 \%$ no consta el teléfono de los pacientes y en el $10,4 \%$ de los casos no se especifica la edad.

Referente a los Datos Formales del Médico, un 6\% no especifican su nombre y apellidos (94\% sí lo hacen, coincidiendo con el estudio de Huertas).Casi un 13\% no indican su número de colegiado o CIAS y un $10,4 \%$ no llevan sello o etiqueta identificativos. Casi la totalidad de los Partes de Interconsulta están firmados.

En total, un 14,9\% de los informes no cuentan con todos los datos completos del médico derivante.

Otros datos administrativos de interés son los siguientes: un 18,9\% de los partes de interconsulta no contienen el nombre del Centro de Salud emisor, un $81,1 \%$ sí lo señalan, porcentaje similar al del estudio de Huertas. Un $86,1 \%$ no especifica el teléfono de contacto del centro. En un 38,8 \% de las peticiones no se especifica si la interconsulta es preferente u ordinaria. Del $61,2 \%$ en las que sí se especifica(123 en total), 67 son preferentes.

La legibilidad es altamente aceptable en los tres apartados referentes a los Datos Formales.

En cuanto a los aspectos clínicos se destacan los siguientes resultados:

Un porcentaje bastante elevado de peticiones no describen la enfermedad actual del paciente: 41,5\% (58,5\% sí lo hacen, porcentaje semejante a Ruiz:58,1\%) (5). En el estudio de García-Campayo (7) de 1993 no había información clínica en un 24\% de los casos. En Huertas (2), entre un 63 y un $66 \%$ tampoco la especificaban.

Un $88,6 \%$ de los PIC especifican el Motivo de Interconsulta -porcentaje similar a lo hallado por Mimbela (4), un 11,4\% no lo concretan -en el estudio de García Campayo (7) eran un 19,5\%-. En Ruiz (5) sólo 37,2\% lo señalan. 
Dentro de los motivos para hacer la interconsulta $65,2 \%$ de las peticiones sólo señalan un motivo, un $20,9 \%$, dos y un $2 \%$, tres motivos.

El 38,8\% especifica Antecedentes Orgánicos y sólo un 7,5\% los tratamientos farmacológicos para estas patologías.

Los antecedentes psiquiátricos personales están presentes en el 39,8\% de los partes de interconsulta, un porcentaje semejante al de algunos estudios (Mimbela:35,09\%) y muy superior al de otros (García Campayo, 6\%; Huertas entre 17 y 26\%).

Especifican si han recibido tratamiento psicofarmacológico anterior en Atención Primaria un 52,7\% de los casos. En el estudio de Ruiz este porcentaje era superior: $75,1 \%$.

En la emisión del juicio diagnóstico nos encontramos cifras en algunos casos similares a lo señalado por otros autores (García Campayo) y en otros casos inferiores (Ruiz, García-Testal o Mimbela). En un 49,8\% de los informes no se indica la observación diagnóstica del FAP (48\% en García Campayo). En García-Testal (3) y Ruiz (5) alrededor de un $20 \%$ son los que no contienen el juicio diagnóstico.

Datos psicosociales de distinta índole son especificados en un 39,3\% de los casos.

La legibilidad en todos los apartados es alta y alcanza porcentajes cercanos al $90-95 \%$, al igual que en otros estudios en los que también ha sido estudiada esta variable (Huertas).

\section{Discusión}

El estudio que se ha llevado a cabo se inscribe dentro de la línea investigadora de un grupo de investigaciones que se han hecho en los últimos años, que han cosechado distintos resultados en función de las características de los métodos utilizados, las variables medidas y las características de la zona (4).

Según Ocio, hay una progresiva adecuación de los servicios de Salud Mental a la realidad de la población, y la derivación entre los niveles asistenciales ha mejorado en los últimos años. No obstante, nuestros datos nos indican que esta mejoría debe seguir produciéndose.

La muestra utilizada en este estudio es semejante a la de otros que se han revisado (Mimbela n=265;García Campayo n=200;Ruiz n=209; Ocio n=147).

La hoja de interconsulta es el medio más utilizado para intercambiar la información, en detrimento de otros como la consulta telefónica, etc. Es por esto que elevando su calidad se podrá mejorar también la comunicación entre atención primaria y especializada. (7).

Esta calidad depende de muchos factores, entre otros las actitudes y motivación de los profesionales. Es importante, como señalan Huertas o Ruiz, implicar a los médicos especialistas, para consensuar los criterios de derivación entre ellos y la literatura existente, en un documento estructurado en apartados mínimos que describan el proceso de la enfermedad. Así se estimularía su cumplimiento.

Asímismo es fundamental implantar sistemas de trabajo que favorezcan la relación interprofesional y la intercomunicación entre los profesionales de los dos niveles, 
a través de elaboración de protocolos y proyectos conjuntos, reuniones, sesiones de formación, discusión de casos clínicos, interconsultas únicas $(3,8,9), \ldots$ para mejorar el seguimiento y tratamiento de los pacientes y en definitiva, la calidad asistencial $(4,10)$. Sería recomendable que en las Unidades de Salud Mental se implantasen métodos de evaluación de su proceso de derivación para contribuir también a esta mejora, como señala Ruiz.

Hemos comprobado un alto porcentaje en la cumplimentación incompleta de los datos referentes a los pacientes, lo que puede ser debido a la utilización de criterios muy exigentes, como señala Huertas en su estudio de 1996. En cualquier caso es llamativo que datos como el teléfono del paciente, básico para poder ponernos en contacto con él, o la edad, sean datos que se omitan en los porcentajes ya indicados más arriba.

Llama la atención asimismo la frecuencia de PIC que no tienen todos los datos completos del médico, sobre todo su número de colegiado o el sello. Podemos explicarlo basándonos en el hecho de que algunos partes proceden de médicos sustitutos o residentes de familia que no cuentan con sello o etiquetas identificativas.

En cuanto a los datos formales es destacable el porcentaje significativo de partes de interconsulta en los que no aparece el teléfono de contacto del centro de Salud que deriva. No obstante, son datos con los que la administrativa del centro cuenta, pero si añadimos que en casi un 20\% no aparece el nombre del centro de salud en cuestión, la labor administrativa sí puede dificultarse considerablemente.

En casi un $40 \%$ de los casos los médicos de primaria no especifican el tipo de interconsulta que realizan, si es preferente, y por lo tanto necesario que sea atendida en un corto espacio de tiempo, u ordinaria, con lo que puede esperar un poco más. Este aspecto también dificulta la labor asistencial de las unidades de salud mental. Un estudio que ampliaría el presente en esta línea sería el de comprobar en qué medida las derivaciones preferentes son consideradas de la misma manera por los profesionales del segundo nivel de atención, teniendo en cuenta criterios de gravedad, etc.

En otros estudios ya se había concluido que los médicos generales recogen de forma muy incompleta datos clínicos importantes como sintomatología, antecedentes psiquiátricos o tratamientos farmacológicos previos $(5,7)$.

En nuestro estudio se corrobora de nuevo, con datos como los expuestos referentes al elevado porcentaje de peticiones en las que no consta la enfermedad actual $(41,5 \%)$, los antecedentes psiquiátricos $(60,2 \%)$ o el tratamiento farmacológico anterior $(47,3 \%)$.

Es preciso en este punto tener en cuenta la presión asistencial a la que se ven sometidos los facultativos de atención primaria (3), lo que sin duda contribuye a la falta de cumplimentación de algunos datos, así como la subjetividad de la sintomatología del paciente, que hacen que las Interconsultas a los servicios de salud mental sean unas de las que más inseguridad causan a estos profesionales a la hora de plasmar el diagnóstico $(3,11)$. 
INFORMES

Hacer una demanda específica de consulta ayuda a integrar a los profesionales de atención primaria en un equipo de salud mental multidisciplinar, ya que son ellos quienes, tras captar, evaluar y manejar inicialmente a su paciente y/o derivarlo, tienen a priori más información que otros profesionales para establecer las dificultades en el manejo del paciente que originan su derivación, aspectos de diagnóstico o en necesidades del tratamiento, sin que ello condicione a otros profesionales del equipo de salud en su valoración (5). En nuestros datos el porcentaje de cumplimentación de esta variable es superior al encontrado en la literatura.

Entre los motivos de interconsulta que se especifican están los siguientes: valoración, con un 58\% (García Campayo 34\%), derivación/seguimiento, con un 10\% (García Campayo 41,5\%), tratamiento específico o concreto,30\% (García Campayo $5 \%$ ), tratamiento farmacológico o control de la medicación un 6\%.Otros motivos (consultar, informes...) ocupan un $11,4 \%$ de los informes que tienen el motivo de interconsulta especificado.

Es muy importante la emisión de juicios diagnósticos desde atención primaria. En nuestro estudio sólo la mitad de los médicos lo hacen, esta valoración inicial puede servir de orientación sobre la evolución de algunos enfermos, aunque sea erróneo al principio. En esta línea se encuentran estudios como el de García Testal (3) sobre la coincidencia entre los diagnósticos de ambos niveles asistenciales.

La atención integral a los problemas de salud mental pasa por tener en cuenta la naturaleza biopsicosocial de los mismos (12). En un 39,3\% de los casos se hacen constar datos psicosociales en nuestro estudio, dato bastante aceptable, y que nos habla de una cierta conciencia en los FAP del modelo integrador biopsicosocial, que tiene en cuenta estos datos además de los clínicos para entender de una forma global al paciente. No se contemplan este tipo de datos en los estudios revisados.

Hay pocas referencias en la literatura en lo que se refiere a la intervención en la mejora de la calidad de la hoja de interconsulta a través de la información fuera del ámbito hospitalario. Serían necesarios más estudios en la línea del de Huertas, que aborda este punto, que excede los límites del nuestro, pero que podría ser planteado en un futuro.

El presente estudio podría ser ampliado con una especificación más concreta de los datos comparando centros de salud del mismo área geográfica o estableciendo un perfil de variables más significativas que sean criterios de calidad de esta información.

\section{Agradecimientos}

Agradezco la colaboración del personal de la Unidad de Docencia e Investigación de la Gerencia de Atención Primaria de Mallorca (especialmente de Magdalena Esteve y Elena Pastor) así como de los compañeros y compañeras de la Unidad de Salud Mental Emili Darder. 
Tabla 1. Frecuencia y porcentajes de los partes de interconsulta enviados por cada centro de salud.

\begin{tabular}{|l|c|c|}
\hline CENTRO DE SALUD & Frecuencias & Porcentajes(\%) \\
\hline 1. Pere Garau & 32 & 16,0 \\
2. Emili Darder & 24 & 12,0 \\
3. Son Gotleu & 45 & 22,5 \\
4. Coll d'en Rebassa & 37 & 18,5 \\
5. Son Ferriol & 9 & 4,5 \\
6. Llucmajor & 20 & 10,0 \\
7. Arenal & 21 & 10,5 \\
8. Trencadors & 12 & 6,0 \\
Sistema & 1 & \\
\hline TOTAL & 201 & 100,0 \\
\hline
\end{tabular}

Tabla 2. Porcentajes obtenidos en cada variable de la hoja de datos. Muestra $n=201$

\begin{tabular}{|c|c|c|c|}
\hline VARIABLES & \multicolumn{2}{|c|}{ 1=CONSTA. $(\%)$} & $0=\mathrm{NO}$ CONSTA $(\%)$ \\
\hline DATOS FORMALES & & \\
\hline DEL PACIENTE & & & \\
\hline - Nombre y apellidos & \multicolumn{2}{|l|}{100,0} & 0 \\
\hline - Teléfono & \multicolumn{2}{|l|}{84,6} & 15,4 \\
\hline - N. ${ }^{\circ}$ de Ident. Personal & \multicolumn{2}{|l|}{86,1} & 13,9 \\
\hline - Edad o fecha de nacimiento & \multicolumn{2}{|l|}{89,6} & 10,4 \\
\hline - Datos completos & \multicolumn{2}{|l|}{72,1} & 27,9 \\
\hline - Legibilidad & \multicolumn{2}{|l|}{99,5} & 0,5 \\
\hline \multicolumn{4}{|l|}{ DEL MÉDICO } \\
\hline - Nombre y apellidos & \multicolumn{2}{|l|}{94,0} & 6,0 \\
\hline - Número de colegiados & \multicolumn{2}{|l|}{87,1} & 12,9 \\
\hline - Sello o etiqueta & \multicolumn{2}{|l|}{89,6} & 10,4 \\
\hline - Firma & \multicolumn{2}{|l|}{99,5} & 0,5 \\
\hline - Datos completos & \multicolumn{2}{|l|}{85,1} & 14,9 \\
\hline • Legibilidad & \multicolumn{2}{|l|}{96,0} & 4,0 \\
\hline OTROS DATOS & $1=\operatorname{CONSTA}(\%)$ & $0=\operatorname{NO} \operatorname{CONSTA}(5)$ & 2=NO PROCEDE $(\%)$ \\
\hline - Centro de salud & 81,1 & 18,9 & \\
\hline - Teléfono de contacto & 13,9 & 86,1 & \\
\hline - Fecha de derivación & 92,5 & 7,5 & \\
\hline - Especifica tipo de intercons. & 61,2 & 38,8 & \\
\hline • Petición completa & 50,2 & 33,8 & 15,9 \\
\hline - Legibilidad & 83,1 & 0 & 16,9 \\
\hline
\end{tabular}


INFORMES

\begin{tabular}{|l|l|l|}
\hline ASPECTOS CLÍNICOS & 1=CONSTA. (\%) & $0=$ NO CONSTA (\%) \\
\hline ENFERMEDAD ACTUAL & 58,5 & 41,5 \\
MOTIVO DE INTERCONS. & 88,6 & 11,4 \\
• Valoración & 57,7 & 42,3 \\
• Derivación/Seguimiento & 10,0 & 90,0 \\
• Tratamiento concreto & 29,9 & 70,1 \\
• Revisar ttmto. farmacológico & 6,0 & 94,0 \\
• Otros (informes, citas...) & 11,4 & 86,6 \\
ANTECEDENTES ORG. & 38,8 & 61,2 \\
TRATAMIENTO ORG. & 7,5 & 92,5 \\
& & \\
ANTECEDENTES PSIQ. & 39,8 & 60,2 \\
TRATAMIENTO PRES. EN A.P. & 52,7 & 47,3 \\
• Duración & 9,5 & 90,5 \\
• Respuesta (fav. o desfav.) & 18,9 & 81,1 \\
DIAGNÓSTICO DESDE A.P. & 50,2 & 49,8 \\
DATOS PSICOSOCIALES & 39,3 & 60,7 \\
LEGIBILIDAD & 94,5 & 5,5 \\
\hline
\end{tabular}

A.P.=Atención Primaria; ttmto=tratamiento

Figura 1. Hoja de Recogida de Datos

Número de caso:

Fecha evaluación PIC:

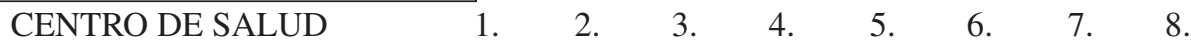
ES UN PIC CON NUEVO FORMATO

1. SI

$0 . \mathrm{NO}$

DATOS FORMALES

DEL PACIENTE

- Nombre y apellidos

1. CONSTA

0. NO CONSTA

- Teléfono

1. CONSTA

0 . NO CONSTA

- DNI/Cód. Ident. pers. o N. ${ }^{\circ}$ S.S.

1. CONSTA

0 . NO CONSTA

- Edad o fecha de nacimiento

1. CONSTA

0 . NO CONSTA

- Completo

1. SI

0 . NO

- Legibilidad

1. SI

$0 . \mathrm{NO}$

DEL MÉDICO

- Nombre y apellidos

1. CONSTA

0. NO CONSTA

- N. ${ }^{\circ}$ Colegiado/CIAS

1. CONSTA

0 . NO CONSTA

- Sello o etiqueta

1. CONSTA

0 . NO CONSTA

- Firma

1. CONSTA

0 . NO CONSTA

- Completo

1. SI

0 . NO

- Legibilidad

1. SI

0 . NO 
INFORMES

OTROS DATOS

- Centro de Salud

1. CONSTA

0. NO CONSTA

- Teléfono de contacto

1. CONSTA

0 . NO CONSTA

- Fecha de derivación

1. CONSTA

0 . NO CONSTA

- Se especifica si la inter. es pref. u ordinaria

1. SI

$0 . \mathrm{NO}$

- ¿El PIC está comp.? (tiene las hojas aut.)

1. SI

0. NO

2. NO PROCEDE

- Legibilidad

1. SI

0. NO

2. NO PROCEDE

ASPECTOS CLÍNICOS

- Enfermedad actual

1. CONSTA

0. NO CONSTA

- Motivo de la interconsulta

1. CONSTA

0 . NO CONSTA

Si consta es:

- Valoración

1. SI

$0 . \mathrm{NO}$

- Derivación-seguimiento

1. SI

$0 . \mathrm{NO}$

- Trat. concreto /farmacol., psicoterapeut.)

1. SI

$0 . \mathrm{NO}$

- Revisar tratamiento farmacológico

1. SI

$0 . \mathrm{NO}$

- Otros

1. SI

$0 . \mathrm{NO}$

- Antecedentes orgánicos

1. CONSTA

0 . NO CONSTA

- Tratamientos

1. CONSTA

0 . NO CONSTA

- Antecedentes psiquiátricos

1. CONSTA

0 . NO CONSTA

- Tratamiento prescrito en Atención Prim.

1. CONSTA

0 . NO CONSTA

- Duración del tratamiento

1. CONSTA

0 . NO CONSTA

- Se indica respuesta al tratamiento

1. CONSTA

0 . NO CONSTA

- Diagnóstico atención primaria

1. CONSTA

0. NO CONSTA

- Datos psicosociales

1. CONSTA

0 . NO CONSTA

- Legibilidad

1. SI

0. NO

Figura 2. Tabla de porcentajes de derivaciones hechas por cada uno de los centros de salud.

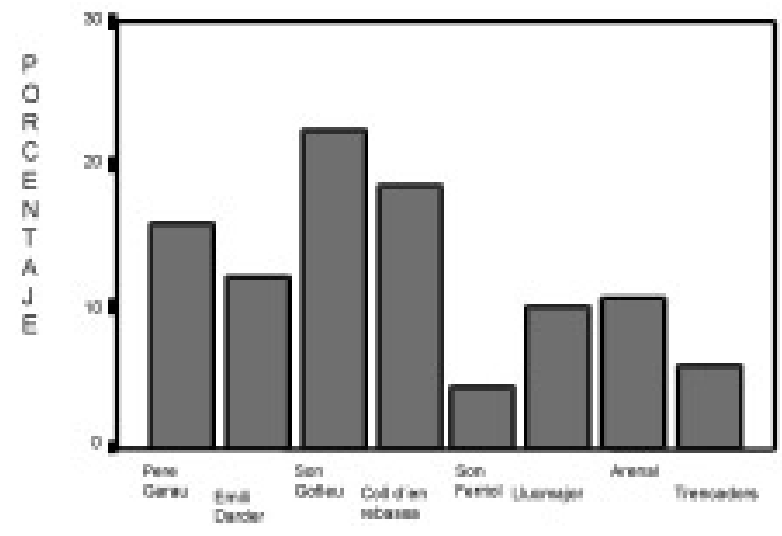




\section{BIBLIOGRAFÍA}

1. Alonso, J.P.; Febrel, M.; Huelin, J., "Factores asociados a la derivación inadecuada entre atención primaria y especializada: estudio cualitativo en médicos de atención primaria", Gac Sanit, 2000, 14 (2):122-130.

2. Huertas, I. y otros, "Mejora de la calidad de la hoja de interconsulta a través de la información", Aten Primaria, 1996, 17 (5):317-320.

3. García-Testal, A. y otros, "Estudio de las derivaciones de atención primaria a salud mental:¿qué coincidencia existe entre los motivos de derivación y el diagnóstico del especialista?", Aten Primaria, 1998, 22 (4):233-238.

4. Mimbela, M.M.; Foradada, S., "Análisis de la interrelación atención primaria-atención especializada en la derivación a pacientes", Aten Primaria, 1993, 12 (2):65-68.

5. Ruiz, F.J.; Piqueras, V.; Aznar, J.A., “¿Es posible mejorar la asistencia psiquiátrica a través del proceso de derivación?", Aten Primaria , 1995, 15 (8):491-498.

6. Ocio, S.; Hernández, M.J., "Perfil de la derivación a Salud Mental. Estudio evolutivo", Interpsiquis, 2002.

7. García Campayo, J.; Sanz, C.; Jimeno, A., "Interconsulta de atención primaria/psiquiatría: utilización de la consulta única”, Aten Primaria, 1993, 13 (2):52-55.

8. Irazábal, L.; Gutiérrez, B., “¿Funciona la comunicación entre los niveles primario y secundario?", Aten Primaria, 1996, 17 (6):376-381.

9. Vargas, B.E. y otros., "Un nuevo modelo de salud mental en atención primaria",(Artículo especial), Aten Primaria, 1997, 20 (6):333-337.

10. Tizón, J.L., "Notas sobre la relación de apoyo y colaboración entre los equipos de atención primaria y los equipos de salud mental", Aten Primaria, 1995, 16 (2):105-114.

11. Chocrón, L. y otros., "Diagnóstico de trastornos psiquiátricos por el médico de atención primaria”, Aten Primaria, 1996, 18 (1):22-26.

12. Asociación Madrileña de Salud Mental., "La atención a la salud mental en Madrid, hoy". Rev Asoc.Esp.Neuropsiq, 1999, XIX, nº 70:337-349.

* Residente de Psicología (PIR) adscrita al Hospital Son Dureta de Palma de Mallorca.

Dirección de contacto: Gerencia de Atención Primaria de Mallorca

Gabinete técnico

Reina Esclaramunda, 9

07003 Palma de Mallorca

Teléf: 971-17 $5884 \quad$ Fax: 971-17 5888

Teléfono particular de la autora: 606689234

Email: pilarpir@hotmail.com

Fecha de recepción: 02-09-02 